\title{
Четыре следования, три порядка, две матрицы, одна бирешетка
}

\author{
Л. Ю.ДЕвяткин
}

\begin{abstract}
In this paper it is shown how four consequence relations defined in terms of designated and anti-designated values allow to produce a six-element bilattice on the basis of two arbitrary finite-valued logical matrices for a propositional language $L$.

Keywords: product of logical matrices, anti-designated values, consequence relation
\end{abstract}

В настоящей работе будет показано, как основе четырех попарно различных отношений логического следования, которые можно определить в терминах выделенных и анти-выделенных значений, и двух произвольных конечнозначных матриц для некоторого пропозиционального языка $L$ можно построить шестиэлементную бирешетку с порядками по отношению логического следования и классу тавтологий.

ОПРЕДЕЛЕНИЕ 1. Пусть дан пропозициональный язык $L$. Логическая матрица $\mathfrak{M}=\langle\mathcal{A}, D>$, где $\mathcal{A}$ есть некоторая алгебра, а $D$ выделенное подмножество множества-носителя $\mathcal{A}$, является матрицей для $L$, е.т.е. для каждого $n$ число $n$-арных базовых операций $\mathcal{A}$ равно числу $n$-арных связок в $L$.

Тогда можно установить взаимно-однозначное соответствие между связками из $L$ и операциями из $\mathcal{A}$ соответствующей местности и определить оценку $L$-формулы (далее - «формулы») $A$ в $\mathfrak{M}$ следующим образом:

ОПРЕДЕЛЕНИЕ 2. Оценка $v$ формулы $A$ в $\mathfrak{M}$ есть отображение $L$ на $\mathcal{A}$, такое что

- если $A$ есть пропозициональная переменная, то $v(A) \in V$, где $V$ есть множество-носитель $\mathcal{A}$; 
- если $A_{1}, A_{2}, \cdots, A_{n}$ есть формулы, и $\mathbb{C}$ есть $n$-арная связка из $L$, то $v\left(\mathbb{C}\left(A_{1}, A_{2}, \cdots, A_{n}\right)\right)=f^{n}\left(v\left(A_{1}\right), v\left(A_{2}\right), \cdots, v\left(A_{n}\right)\right)$, где $f^{n}$ есть операция из $\mathcal{A}$, соответствующая $\mathbb{C}$.

ОПРЕДЕЛЕНИЕ 3. Формула $A$ логически следует из множества формул $\Gamma$ в $\mathfrak{M}(\Gamma \vDash(\mathfrak{M}) B)$, е.т.е. не существует оценки $v$ в $\mathfrak{M}$, такой что $v[\Gamma] \subseteq D(\mathfrak{M})$ (то есть, каждая формула из $\Gamma$ принимает значение, выделенное в $\mathfrak{M})$ и $v(A) \notin D(\mathfrak{M})$.

ОПРЕДЕЛЕНИЕ 4. Формула $A$ является тавтологией в $\mathfrak{M}(\vDash$ $(\mathfrak{M}) B$ ), е.т.е. $A$ логически следует из любого (в том числе пустого) множества формул $\Gamma$ в $\mathfrak{M}$.

Обозначим как $C(\mathfrak{M})$ множество упорядоченных пар $\langle\Gamma, B>$, таких что $Г$ есть множество формул, $B-$ формула и $\Gamma \vDash(\mathfrak{M}) B$. Обозначим как $T(\mathfrak{M})$ множество тавтологий $\mathfrak{M}$. Ясно, что каждая пара $<F m, \vDash(\mathfrak{M})>$, где $F m$ есть множество формул языка $L$, a $\vDash(\mathfrak{M})$ есть отношение логического следования, заданное на $F m$, порождает собственные классы $C(\mathfrak{M})$ и $T(\mathfrak{M})$.

Если $\mathfrak{A}$ и $\mathfrak{B}$ есть матрицы для $L$, можно установить взаимнооднозначное соответствие между их базовыми операциями. Это позволяет определить следующую операцию на $\mathfrak{A}$ и $\mathfrak{B}:$

ОПРЕДЕЛЕНИЕ 5. Будем называть произведением матриц $\mathfrak{A}$ и $\mathfrak{B}(\mathfrak{A} \times \mathfrak{B})$ такую матрицу $\mathfrak{C}$, что

- $V(\mathfrak{C})$ есть прямое произведение $V(\mathfrak{I})$ и $V(\mathfrak{B})$;

- каждой паре соответствующих $k$-арных базовых операций $f^{k}\left(x_{1}, x_{2}, \cdots, x_{k}\right)$ из $\mathfrak{A}$ и $g^{k}\left(y_{1}, y_{2}, \cdots, y_{k}\right)$ из $\mathfrak{B}$ взаимнооднозначно сопоставлена базовая операция $h^{k}$ из $\mathfrak{C}$, причем $h^{k}\left(<\quad x_{1}, y_{1} \quad>,<\quad x_{2}, y_{2} \quad>, \cdots,<x_{k}, y_{k} \quad>\right)=$ $<f^{k}\left(x_{1}, x_{2}, \cdots, x_{k}\right), g^{k}\left(y_{1}, y_{2}, \cdots, y_{k}\right)>$;

- значение $<x_{i}, y_{j}>$ в $\mathfrak{C}$ является выделенным $\left(<x_{i}, y_{j}>\epsilon\right.$ $D(\mathfrak{C}))$, е.т.е. $x_{i} \in D(\mathfrak{H})$ и $y_{j} \in D(\mathfrak{B})$;

- значение $<x_{i}, y_{j}>$ в $\mathfrak{c}$ является анти-выделенным $\left(<x_{i}, y_{j}>\in D^{*}(\mathfrak{C})\right)$, е.т.е. $x_{i} \notin D(\mathfrak{A})$ и $y_{j} \notin D(\mathfrak{B})$.

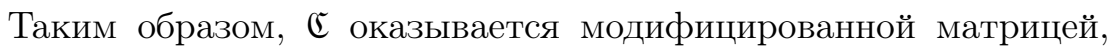
где к классам выделенных и невыделенных значений добавля- 
ется третий - класс анти-выделенных значений, и каждый элемент множества-носителя $\mathfrak{C}$ принадлежит одному из трех классов. Матрицы такого типа называются $q$-матрицами [4]. Можно определить четыре различных отношения логического следования в произвольной $q$-матрице $\mathfrak{\Re}$.

ОПРЕДЕЛЕНИЕ 6.

1. $\Gamma \vDash_{t}(\mathfrak{N}) B$, е.т.е. не существует оценки $v$ в $\mathfrak{N}$, такой что $v[\Gamma] \subseteq D(\mathfrak{N})$ и $v(A) \notin D(\mathfrak{N})$ (классическое следование);

2. $\Gamma \vDash_{f}(\mathfrak{N}) B$, е.т.е. не существует оценки $v$ в $\mathfrak{N}$, такой что $v[\Gamma] \subseteq \overline{D^{*}(\mathfrak{N})}$ (то есть, ни одна из формул не принимает анти-выделенное в $\mathfrak{N}$ значение) и $v(A) \in D^{*}(\mathfrak{R})$ (см. [1]);

3. $\Gamma \vDash_{q}(\mathfrak{N}) B$, е.т.е. не существует оценки $v$ в $\mathfrak{N}$, такой что $v[\Gamma] \subseteq \overline{D^{*}(\mathfrak{N})}$ и $v(A) \notin D(\mathfrak{N})(q$-следование, $[4]) ;$

4. $\Gamma \vDash_{p}(\mathfrak{N}) B$, е.т.е. не существует оценки $v$ в $\mathfrak{N}$, такой что $v[\Gamma] \subseteq D(\mathfrak{N})$ и $v(A) \in D^{*}(\mathfrak{N})(p$-следование, $[2])$.

Каждое из этих отношений характеризует собственные классы формул $C^{i}(\mathfrak{N})$ и $T^{i}(\mathfrak{N})(i \in\{t, f, q, p\})$ языка, матрицей для которого является $\mathfrak{A}$. В матрицах, где $D \cap D^{*}=\varnothing$, между полученными отношениями следования имеет место порядок по $\subseteq$, где $\vDash_{q}$ есть минимум, а $\vDash_{p}$ есть максимум [6]:

УТВЕРЖДЕНИЕ 1.

i. $C^{q}(\mathfrak{N}) \subseteq C^{t}(\mathfrak{N})$

ii. $C^{q}(\mathfrak{N}) \subseteq C^{f}(\mathfrak{N})$;

iii. $C^{t}(\mathfrak{N}) \subseteq C^{p}(\mathfrak{N})$;

iv. $C^{f}(\mathfrak{N}) \subseteq C^{p}(\mathfrak{N})$.

Ясно, что Утверждение 1 выполняется для $\mathfrak{C}=(\mathfrak{A} \times \mathfrak{B})$. Аналогичный порядок имеет место между $C(\mathfrak{H}), C(\mathfrak{B}), C^{q}(\mathfrak{C})$ и $C^{p}(\mathfrak{C})$.

УТВЕРЖДЕНИЕ 2. Пусть $\mathfrak{c}=(\mathfrak{A} \times \mathfrak{B})$. Тогда имеет место следуюшее: 

i. $C^{q}(\mathfrak{C}) \subseteq C(\mathfrak{H})$
ii. $C^{q}(\mathfrak{C}) \subseteq C(\mathfrak{B})$;
iii. $C(\mathfrak{A}) \subseteq C^{p}(\mathfrak{C})$;
iv. $C(\mathfrak{B}) \subseteq C^{p}(\mathfrak{C})$

Доказательство. (i) Пусть $\Gamma \vDash_{q}(\mathfrak{C}) B$ и $\Gamma \not \models(\mathfrak{U}) B$. Тогда существует оценка $v$ в $\mathfrak{A}$, такая что $v[\Gamma] \subseteq D(\mathfrak{A})$ и $v(A) \notin D(\mathfrak{A})$. В этом случае, по определению $\mathfrak{c}, w[\Gamma] \subseteq \overline{D^{*}(\mathfrak{C})}$ и $w(A) \notin D(\mathfrak{C})$ при некоторой оценке $w$ в $\mathfrak{C}$. Следовательно $\Gamma \not \not_{q}(\mathfrak{E}) B$, что противоречит условию. Доказательство для (ii) аналогично.

Теперь докажем (iii) $u$ (iv). Пусть $\Gamma \not_{q}(\mathfrak{C}) B$ и $B$ следует из $\Gamma$ в $\mathfrak{A}$ или $\mathfrak{B}$. Тогда существует оценка $w$ в $\mathfrak{C}$, такая что $v[\Gamma] \subseteq D(\mathfrak{C})$ и $v(A) \in D^{*}(\mathfrak{C})$. Это возможно только в том случае, когда имеются оценки $v$ в $\mathfrak{A}$ и $u$ в $\mathfrak{B}$, при которых $v[\Gamma] \subseteq D(\mathfrak{H})$ и $v(A) \notin D(\mathfrak{A})$, а также $u[\Gamma] \subseteq D(\mathfrak{B})$ и $u(A) \notin D(\mathfrak{B})$. Однако тогда $\Gamma \not \models(\mathfrak{H}) B$ и $\Gamma \not \models(\mathfrak{B}) B$.

Q.E.D.

Итак, $\vDash_{q}$ снова оказывается минимумом, а $\vDash_{p}-$ максимумом. То есть, из Утверждений 1 и 2 можно сделвать вывод, что имеет место решетка с четырьмя промежуточными элементами. Как выясняется, на данных элементах также можно задать порядок по $\subseteq$, однако на этот раз речь пойдет о классах тавтологий.

УТВЕРЖДЕНИЕ 3. Пусть $\mathfrak{c}=(\mathfrak{A} \times \mathfrak{B})$. Тогда имеет место следующее:
i. $T^{t}(\mathfrak{C}) \subseteq T(\mathfrak{A})$
ii. $T^{t}(\mathfrak{C}) \subseteq T(\mathfrak{B}) ;$
iii. $T(\mathfrak{A}) \subseteq T^{f}(\mathfrak{C})$;
iv. $T(\mathfrak{B}) \subseteq T^{f}(\mathfrak{C})$

Доказательство. В действительности могут быть доказаны более сильные утверждения: (v) $T^{t}(\mathfrak{C})=T(\mathfrak{H}) \cap T(\mathfrak{B}) ;(v i) T^{f}(\mathfrak{C})=$ $T(\mathfrak{H}) \cup T(\mathfrak{B})$. Доказательство $(v)$ представлено в [5]. Докажем 
(vi) (доказательство аналогичного факта в другой формулировке имеется в [5] и [3]).

Пусть формула $A$ принадлежит множеству $T(\mathfrak{H}) \cup T(\mathfrak{B})$. Тогда $A \in T(\mathfrak{I})$ или $A \in T(\mathfrak{B})$ (в неисключающем смысле). Если $A \in T(\mathfrak{U})$, то $v(A) \in D(\mathfrak{H})$ при каждой оценке $v$ в $\mathfrak{A}$. Но тогда, согласно определению $\mathfrak{A} \times \mathfrak{B}$, не существует оценки $w$ в $\mathfrak{C}$, при которой $w(A) \in D^{*}(\mathfrak{C})$. Следовательно, $A \in T^{f}(\mathfrak{C})$. Аналогично для $A \in T(\mathfrak{B})$.

Теперь пусть $A \notin T(\mathfrak{A})$ или $A \notin T(\mathfrak{B})$. Тогда существуют оценка $v$ в $\mathfrak{A}$, такая что $v(A) \notin D(\mathfrak{A})$ и оценка $u$ в $\mathfrak{B}$, такая что $u(A) \notin D(\mathfrak{B})$. Но тогда, по определению $\mathfrak{A} \times \mathfrak{B}$, существует оценка $w$ в $\mathfrak{C}$, при которой $w(A) \in D^{*}(\mathfrak{C})$ и $A \notin T^{f}(\mathfrak{C}) . \quad$ Q.E.D.

Из определений $\vDash_{t}(\mathfrak{N}), \vDash_{f}(\mathfrak{N}), \vDash_{q}(\mathfrak{N}), \vDash_{p}(\mathfrak{N})$, а также определения тавтологии вытекает, что $T^{t}(\mathfrak{M})=T^{q}(\mathfrak{N})$ и $T^{f}(\mathfrak{N})=T^{p}(\mathfrak{N})$.

Таким образом, произвольные логические матрицы $\mathfrak{A}$ и $\mathfrak{B}$ для пропозиционального языка $L$ и их произведение $\mathfrak{c}$ с четырьмя отношениями следования образуют шестиэлементную бирешетку систем $<F m, \vDash(\mathfrak{U})>,<F m, \vDash(\mathfrak{B})>,<F m, \vDash_{t}(\mathfrak{C})>$, $<F m, \vDash_{f}(\mathfrak{C})>,<F m, \vDash_{q}(\mathfrak{C})>$ и $<F m, \vDash_{p}(\mathfrak{C})>$, в которой первый порядок есть порядок по включению класса $C$, а второй порядок - по включению класса $T$.

В заключение отметим одно полезное следствие. Из полученных результатов, в частности, вытекают критерии эквивалентности матриц для $L$ по классу тавтологий и по отношению логического следования: $T(\mathfrak{H})=T(\mathfrak{B})$, е.т.е. $T^{t}(\mathfrak{A} \times \mathfrak{B})=T^{f}(\mathfrak{U} \times \mathfrak{B})$; $C(\mathfrak{H})=C(\mathfrak{B})$, е.т.е. $C^{p}(\mathfrak{H} \times \mathfrak{B})=C^{q}(\mathfrak{H} \times \mathfrak{B})$.

\section{Литература}

[1] Dunn J.M. Partiality and its dual // Studia Logica. V. 66. 2000. Pp. 5-40.

[2] Frankowski S. Formalization of a plausible inference // Bulletin of the Section of Logic. V. 33. 2004. Pp. 41-52.

[3] Kalicki J. A test for the equality of truth-tables // The Journal of Symbolic Logic. V. 17. № 3. 1952. Pp. 161-163.

[4] Malinowski G. Inferential many-valuedness // Philosophical logic in Poland, J. Woleński (ed.). Synthese Library, Kluwer Academic Publishers, Dordrecht. 1994. Pp. 74-84.

[5] Rescher N. Many-Valued Logic. New York (McGraw-Hill), 1969. Reprinted: Aldershot (Gregg Revivals), 1993. Pp. 96-101.

[6] Shramko Y., Wansing H. Entailmant realtions and/as truth values // Bulletin of the Section of Logic. V. 36:3/4. 2007. Pp. 131-143. 